CORRIGENDUM

doi:10.1038/nature11515

\title{
Corrigendum: Mitochondrial DNA that escapes from autophagy causes inflammation and heart failure
}

Takafumi Oka, Shungo Hikoso, Osamu Yamaguchi, Manabu Taneike, Toshihiro Takeda, Takahito Tamai, Jota Oyabu, Tomokazu Murakawa, Hiroyuki Nakayama, Kazuhiko Nishida, Shizuo Akira, Akitsugu Yamamoto, Issei Komuro \& Kinya Otsu

\section{Nature 485, 251-255 (2012); doi:10.1038/nature10992}

In this Letter, several images were mistakenly switched or duplicated during preparation of the artwork. In Figs if and 2a, the shamoperated Dnase $2 a^{-1-}$ and TAC-operated Dnase $2 a^{+1+}$ mice panels were switched. In Fig. $4 \mathrm{~d}$, the panel showing CD3 staining for ODN2088 control-treated TAC-operated Dnase $2 a^{+/+}$mice (now shown correctly as black-bordered panel in Fig. 1 below) is a duplicate of that showing Ly6G staining for ODN2088-treated TAC-operated Dnase $2 a^{-1-}$ mice. The panel showing CD45 staining for ODN2088treated TAC-operated Dnase $2 a^{+/+}$(now shown correctly as bluebordered panel in Fig. 1 below) was prepared from the original picture of ODN2088 control-treated TAC-operated Dnase $2 a^{+++}$. In Supplementary Fig. 4c, sham-operated Dnase $2 a^{-1-}$ and TAC-operated Dnase $2 a^{+1+}$ mice panels were switched. Finally, in Supplementary Fig. 10d, the panels showing CD3 and Ly6G staining for shamoperated $\mathrm{Tlr} 9^{+/+}$mice were switched. These corrections do not alter any of the conclusions of this Letter, and the authors apologize for any confusion these errors may have caused.

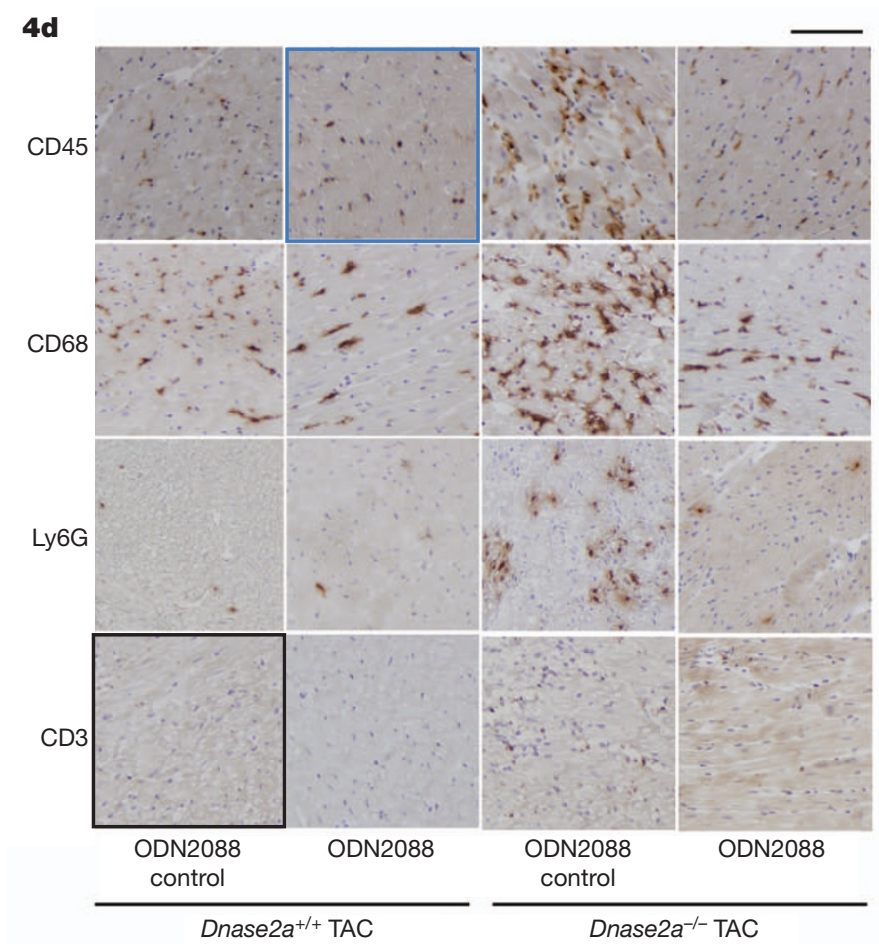

Figure $1 \mid$ This is the corrected Fig. 4d of the original Letter. 